\title{
Turnover of Plasma Cholesterol in Man
}

\author{
DeWrtt S. Goodman and Robert P. Noble \\ From the Department of Medicine, Columbia University College of Physicians \\ and Surgeons, New York, and the Sharon Research Institute, Sharon Hospital, \\ Sharon, Connecticut
}

A в S T к A C T Cholesterol- $4-{ }^{24} \mathrm{C}$ was injected intravenously into a series of normal men, untreated hyperlipidemic patients, and hyperlipidemic patients being treated with cholestyramine. The specific radioactivity of plasma total cholesterol was measured during the ensuing 10 wk. 16 studies were carried out in 10 subjects. Analysis of the turnover curves of plasma cholesterol revealed that in every study the turnover of plasma cholesterol conformed to a two-pool model. Each turnover curve was analyzed in terms of this model, as expressed by the equation: specific activity = $\mathrm{C}_{\mathbf{A}} \mathrm{e}^{-\alpha \mathrm{t}}+\mathrm{C}_{\mathrm{B}} \mathrm{e}^{-\beta \mathrm{t}}$. The parameters which were calculated included the constants $C_{\Delta}, C_{B}, \alpha$, and $\beta$; the size of the first pool $\left(M_{\mathbf{A}}\right)$; the rate constants for the total rate of removal of cholesterol from each pool $\left(k_{A \Delta}\right.$ and $\left.k_{B B}\right)$; and the production rate in pool $A\left(P R_{\Delta}\right)$. In two normal men and five untreated patients the average size of pool $A$ was $25 \mathrm{~g}$.

The effect of cholestyramine was assessed by comparing the results obtained without therapy with those obtained during therapy in five subjects studied under both conditions. Cholestyramine therapy produced a large increase in $\mathrm{PR}_{\mathbf{\Delta}}$ (from 0.98 to $1.98 \mathrm{~g} /$ day) and in the rate of removal of cholesterol from pool A. Cholestyramine did not significantly alter the size of pool A.

It is not possible to calculate the size of the total body exchangeable pool of cholesterol from the turnover curve of plasma cholesterol. It is also not possible to calculate the metabolic turnover rate,

Part of this work was presented at the annual meeting of the American Heart Association in New York, October 1966 (1).

Received for publication 23 March 1967 and in revised form 20 September 1967. i.e., the rate of cholesterol degradation and excretion, in the whole body. This parameter can, however, be estimated by assuming that cholesterol is removed from the body only by way of the tissue pools that comprise pool A. Under these conditions the metabolic turnover rate is equal to the production rate in pool $A$.

\section{INTRODUCTION}

The turnover of plasma cholesterol in man has been studied repeatedly during the past few years (2-7). In these studies, isotopically labeled cholesterol $(3,4,6,7)$ or a biosynthetic precursor of cholesterol (5) was injected intravenously, and the specific radioactivity of plasma cholesterol was then determined during the ensuing weeks. These studies demonstrated that, after isotope administration, the semilogarithmic plot of cholesterol specific radioactivity vs. time describes a curve during the first 4-6 wk. whereas beyond this time the plot is linear. The changing slope of the curve during the first few weeks has been shown to be due to slow rates of equilibration between the cholesterol pools of plasma and various tissues $(8,9)$. Thus, postmortem analyses of tissues of patients who received labeled cholesterol from 1 to 226 days (9) or from 2.5 to 137 days (8) before death demonstrated that complete equilibration between plasma and tissue cholesterol required up to a month for some tissues, and more than a month for arteries. Once equilibration between plasma and tissue cholesterol was achieved, however, it was maintained indefinitely. The cholesterol in all tissues except brain was found to equilibrate with plasma cholesterol (9). On the basis of these results, it has usually been assumed that the late, 
linear portion of the plasma cholesterol turnover curve reflects the turnover of the total body pool of exchangeable cholesterol. Accordingly, in some studies estimates of the turnover of total body cholesterol and of the size of the total body miscible pool of cholesterol have been derived from turnover curves of plasma cholesterol.

The studies reported here were undertaken as part of an extensive investigation on the effects of cholestyramine on the metabolism of plasma lipids. In these studies, ${ }^{14} \mathrm{C}$-labeled cholesterol was injected intravenously into a series of normal men, untreated hyperlipidemic patients, and hyperlipidemic patients being treated with cholestyramine. Analysis of the curves of plasma cholesterol specific radioactivity vs. time revealed that in every instance the turnover of plasma cholesterol conformed to a two-pool model. The purpose of this paper is to present the results of these studies and to discuss in detail the interpretation of plasma cholesterol turnover curves.

\section{METHODS}

10 volunteer subjects, whose ages and sexes are given in Table I, participated in these studies. Two of the subjects (R.N. and R.O.) served as normal controls. The other eight subjects all had mild to moderate degrees of hyperlipidemia (see Table I). Seven of the hyperlipidemic patients (all except H.D.) had clinical coronary heart disease, as indicated by the presence of angina pectoris, or by the past history of documented myocardial infarction. All subjects ate their usual diets throughout the studies. None of the subjects lost or gained significant amounts of weight during the periods of study.

16 turnover studies were carried out in two series (study series I and II), separated by an interval of $1 \mathrm{yr}$. In study series I, three untreated patients and five patients being treated with cholestyramine (Questran, Mead Johnson \& Co.) were injected intravenously with cholesterol- ${ }^{14} \mathrm{C}$. In study series II, two normal controls, two untreated patients, three patients being treated with cholestyramine, and one patient taking ethyl p-chlorophenoxyisobutyrate (CPIB, clofibrate; Atromid-S, Ayerst Laboratories) were studied in a similar fashion. Cholestyramine was administered at a level of $12 \mathrm{~g} /$ day, and CPIB at a dose of $2 \mathrm{~g} /$ day. All subjects studied during periods of drug treatment had taken the drug for 3 or more months before isotope injection. All subjects had stable plasma cholesterol and triglyceride levels for several .weeks before the start of each study and throughout the period of each study. As indicated in Table I, six of the subjects were studied, under different circumstances, in both study series I and II.

In each study series, $500 \mu \mathrm{c}$ of cholesterol-4- ${ }^{14} \mathrm{C}$ ( New
England Nuclear Corp., Boston, Mass.; specific radioactivity $56 \mu \mathrm{c} / \mu \mathrm{mole}$ ) was dissolved in $0.5 \mathrm{ml}$ of acetone, and the acetone solution was then injected slowly, via a $100 \mu 1$ syringe, beneath the surface of $30 \mathrm{ml}$ of serum. The serum was freshly drawn from one of the investigators earlier in the day and was gently swirled during the period of acetone addition. The labeled serum was shaken gently at $37^{\circ} \mathrm{C}$ for $2 \mathrm{hr}$ and at room temperature for an additional 16 to $20 \mathrm{hr}$. The serum was then sterilized by passage through a Millipore filter of pore size $0.2 \mu$.

Serum containing approximately $30 \mu \mathrm{c}$ of cholesterol $-{ }^{14} \mathrm{C}$ was injected intravenously into each subject. The amount injected was measured precisely in each study and varied between 26 and $27 \mu \mathrm{c}$ in study series I and between 33 and $35 \mu \mathrm{c}$ in study series II. Samples of venous blood were collected before breakfast after 1, 3, and 7 days and then at weekly intervals for a total of 9 or 10 wk. In several instances an early sample was also collected $8 \mathrm{hr}$ after isotope injection. Blood cells were sedimented by centrifugation at $2000 \mathrm{rpm}$ for $30 \mathrm{~min}$ at $4^{\circ} \mathrm{C}$. A portion of each plasma sample was collected and stored at $-20^{\circ} \mathrm{C}$, and was later used for the measurement of the plasma concentrations of cholesterol and triglyceride by the methods of Abell, Levy, Brodie, and Kendall (10) and of Van Handel and Zilversmit (11), respectively. Other portions of each plasma sample were added to 2 volumes of ethanol, followed by the addition of $\mathrm{KOH}$ to a final concentration of $3 \%$. The samples were flushed with nitrogen and saponified at $60-70^{\circ} \mathrm{C}$ for 1 to $2 \mathrm{hr}$. The nonsaponifiable lipids were collected by three extractions, each time with an equal volume of hexane. The hexane was evaporated with a stream of nitrogen, and the nonsaponifiable lipid was dissolved in $5.00 \mathrm{ml}$ of benzene. Measured portions of each benzene solution were assayed for ${ }^{11} \mathrm{C}$ in a Packard Tri-Carb liquird scintillation counter, using $0.5 \%$ diphenyloxazole in toluene as scintillation solvent. Other portions of each benzene solution were assayed for cholesterol by the method of Abell et al. (10). The concentrations of plasma cholesterol calculated from the results of these assays agreed closely with the concentrations determined by direct analysis of plasma. The specific radioactivity of cholesterol in each sample was determined from the measured concentration of ${ }^{14} \mathrm{C}$ and of cholesterol in each benzene solution of plasma nonsaponifiable lipids. For purposes of comparison, all results have been adjusted to an injected dose of $30 \mu \mathrm{c}$ of cholesterol- ${ }^{14} \mathrm{C}$.

\section{RESULTS}

Analysis and discussion of plasma cholesterol turnover curves. Fig. 1 shows the results obtained after injecting cholesterol- ${ }^{14} \mathrm{C}$ into normal control subject R.N. The semilogarithmic plot of specific radioactivity vs. time described a curve during the first $5 \mathrm{wk}$, whereas from $6 \mathrm{wk}$ onward the plot followed a straight line. Similar curves were obtained in all of the studies which we have carried 


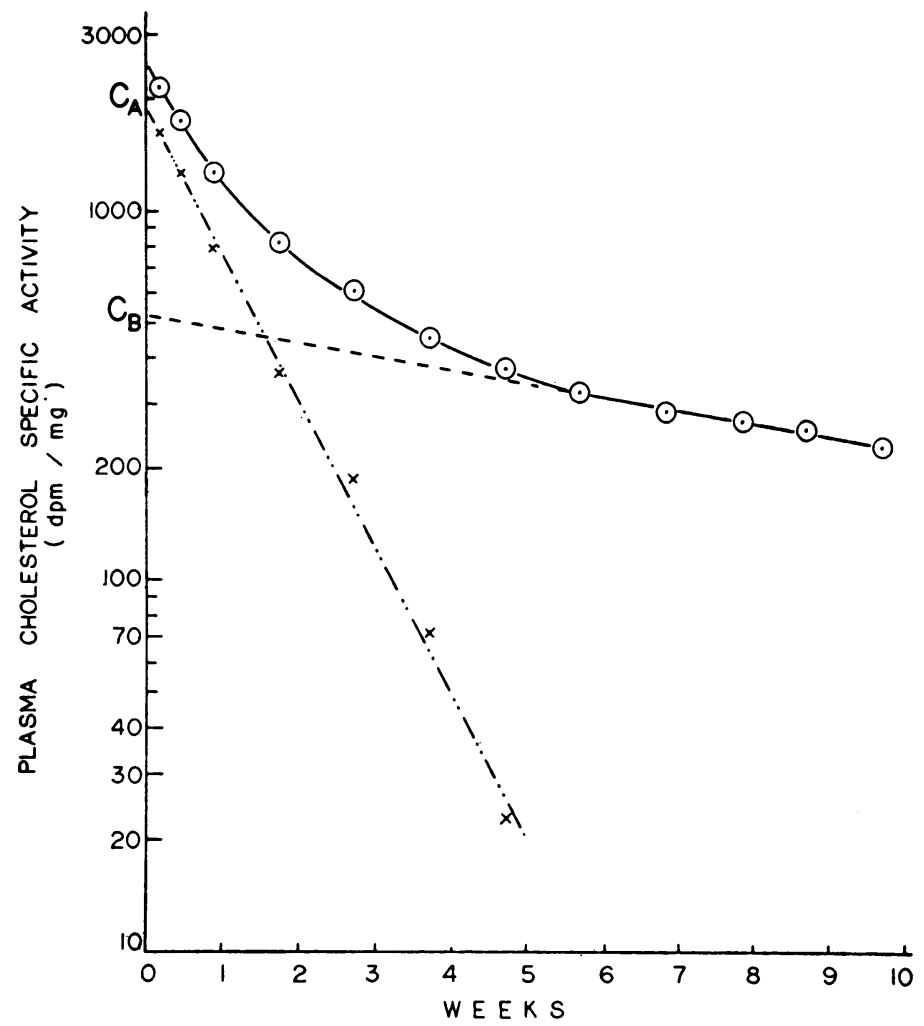

Figure 1 The turnover of plasma cholesterol in normal control subject R.N. The circles, connected by a solid line, represent the experimental values observed during the 10 wk study. Extrapolation of the terminal linear portion of the curve back to zero time (dashed line) provides the intercept $\mathrm{C}_{\mathbf{B}}$. Subtraction of this extrapolated line from the experimental points provides the difference values shown as small crosses, and connected by an interrupted (dash-dot-dot) line.

out; in every study a constant exponential rate of fall was achieved after 5 or $6 \mathrm{wk}$.

Each turnover curve was analyzed in order to determine the number of kinetically distinguishable pools (compartments) involved in the turnover of plasma cholesterol in man. As shown in Fig. 1, this analysis was done by extrapolating the terminal linear portion of the turnover curve back to zero time. From each experimental point measured during the first $5 \mathrm{wk}$ the value of the corresponding point on the extrapolated line was then subtracted, and the difference values so obtained were plotted semilogarithmically. As shown in Fig. 1, the plot of the difference between the experimental points and the extrapolated line described a straight line, indicating that the turnover of plasma cholesterol conformed to a two-pool model (12. 13). If more than two kinetically distinguishable pools had been involved in the turnover of plasma cholesterol, the first portion of the plot of the difference would have described a curve rather than a straight line, and it would have been necessary to continue the process of subtraction in order to determine the number of pools involved.

Similar analyses were carried out in each of the
16 studies resported here. In every instance the results conformed to a two-pool model.

A detailed discussion of the kinetic analysis of two-pool systems was recently reported by Gurpide, Mann, and Sandberg (13). As noted by these workers, the general two-pool system can be described by the model shown in Fig. 2. In this model the specific activity of the labeled substance in pool $\mathrm{A}$, subsequent to its initial injection into pool $\mathrm{A}$, can be characterized by the equation:

$$
a=C_{A} e^{-\alpha t}+C_{B} e^{-\beta t}
$$

in which $\mathrm{a}=$ specific activity in pool $\mathrm{A}$ of the substance under study; $C_{\mathbf{A}}, C_{B}, \alpha$, and $\beta$ are constants (see below); e is the base of the natural logarithms; and $\mathrm{t}=$ time. This model and this equa-

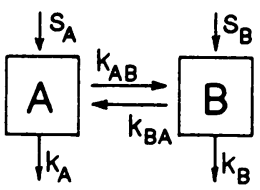

Figure 2 General two-pool ( $\mathrm{A}$ and $\mathrm{B}$ ) model. Rate constants are denoted by the $k$ values; $s_{A}$ and $s_{B}$ are the rates of entry of material into the pools from outside the system. [After Gurpide et al. (13)].

Turnover of Plasma Cholesterol in Man 
tion satisfactorily characterize turnover curves of plasma cholesterol in man, such as the one shown in Fig. 1.

A number of kinetic and other parameters can be determined from a plasma cholesterol turnover curve such as that shown in Fig. 1 (see reference 13 for a complete discussion). First of all, the $y$-intercepts of the two straight lines directly provide the values of the constants $C_{A}$ and $C_{B}$ in equation (1) (see Fig. 1). Second, the constants $\alpha$ and $\beta$ can be directly calculated from the slopes of the two straight lines. Thus the values of these constants are determined from the half-lives of the two exponentials, since $\alpha=\ln 2 / \mathrm{t}_{1}=0.69315 / \mathrm{t}_{3}$ (of the first exponential), and $\beta=\ln 2 / \mathrm{t}_{1}$ (of the second exponential). Third, the size of the first pool (pool A) can be calculated as follows:

$$
M_{A}=\frac{R_{A}}{C_{A}+C_{B}}
$$

where $M_{A}=$ the size of pool $A$, and $R_{A}=$ the amount of isotope injected into pool A. Fourth, the rate constants for the total rate of removal of cholesterol from pool $A\left(k_{\mathbf{A} \mathbf{A}}\right)$ and from pool $B$ $\left(k_{B B}\right)$ can be calculated as follows :

$$
\begin{gathered}
k_{A A}=\frac{-\alpha M_{A} C_{A}-\beta M_{A} C_{B}}{R_{A}} \\
k_{B B}=-\left(\alpha+\beta+k_{A A}\right) .
\end{gathered}
$$

Finally, it is possible to calculate the production rate of cholesterol in pool $\mathrm{A}\left(\mathrm{PR}_{\mathbf{A}}\right)$. The production rate in a given pool has been said to represent the rate of entry of material into the pool, excluding recycled material originating in that pool (13, 14). For the turnover of plasma cholesterol, using the two-pool model under discusion:

$$
P R_{A}=\frac{R_{A} \alpha \beta}{\alpha C_{B}+\beta C_{A}} .
$$

As indicated by Gurpide et al. (13), other parameters describing the two-pool model (Fig. 2) cannot be derived from turnover curves of the type shown in Fig. 1. It is, for example, not possible to determine the size of the second pool (pool B) except for the very special case where either $\mathbf{s}_{\mathbf{A}}$ and $\mathbf{k}_{\mathbf{A}}$ both equal zero, or $\mathrm{s}_{\mathbf{B}}$ and $\mathrm{k}_{\mathbf{B}}$ both equal zero. It is extremely unlikely that these special conditions apply. to the turnover of cholesterol (see Discussion). It is hence not possible to determine the size of the total body exchange- able cholesterol (pool A + pool B) from these turnover data. It is also not possible to determine precisely the values of the individual rate constants shown in Fig. 2, except for the special case where either $k_{A}$ or $k_{B}$ equals zero. If, however, one assumes that $k_{B}$ approximates zero, then $k_{1 B_{A}}=-$ $\mathrm{k}_{\mathrm{BB}} ; \mathrm{k}_{\mathrm{A}}=\alpha \beta / \mathrm{k}_{\mathrm{BA}}$; and $\mathrm{k}_{\mathrm{AB}}=-\mathrm{k}_{\mathrm{AA}}-\mathrm{k}_{\mathrm{A}}$ (see reference 13 for derivations).

Experimental results. Table I presents the values for each of the several parameters discussed above for each of the 16 turnover studies carried out. It should be noted that the actual. experimentally obtained turnover curves can readily be constructed from the values of $C_{A}, C_{B}$. and the half-lives of the two exponentials.

Computer calculations. The results presented in Table I were obtained by the hand fitting of the data from each study to a sum of two exponentials. In order to evaluate the precision of these results, the data obtained in six of the 16 studies were also subjected to compartmental analysis by a digital computer, as described by Berman and his colleagues $(12,15-17)$. These calculations were kindly carried out by Dr. Arthur Frank, in collaboration with Doctors Daniel Steinberg and Mones Berman, at the National Institutes of Health, Bethesda, Md. The results of these analyses are presented in Table II. Comparison of the values in Table II with the corresponding values in Table I indicates that the results obtained by hand fitting the data (Table I) agree very closely with those obtained by computer analysis. The computer analysis indicates that the parameters are definable with a fairly high degree of precision, since the standard deviations for the various parameters were found to be small in all instances (see Table II). The computer analysis also confirmed the fact that the two-compartment model provides an excellent fit for the data obtained in these studies.

Effect of cholestyramine. The effect of cholestyramine was assessed by comparing the results obtained without therapy with those obtained during cholestyramine therapy in the five subjects (H.D., M.H., L.L., H.K., and L.P.) who were studied under both conditions. Cholestyramine therapy, at a dose level of $12 \mathrm{~g} /$ day, produced an average lowering of the plasma cholesterol level of $14 \%$ (range 28 to $-2 \%$ ). Similar results have been observed with a larger series of 12 pa- 


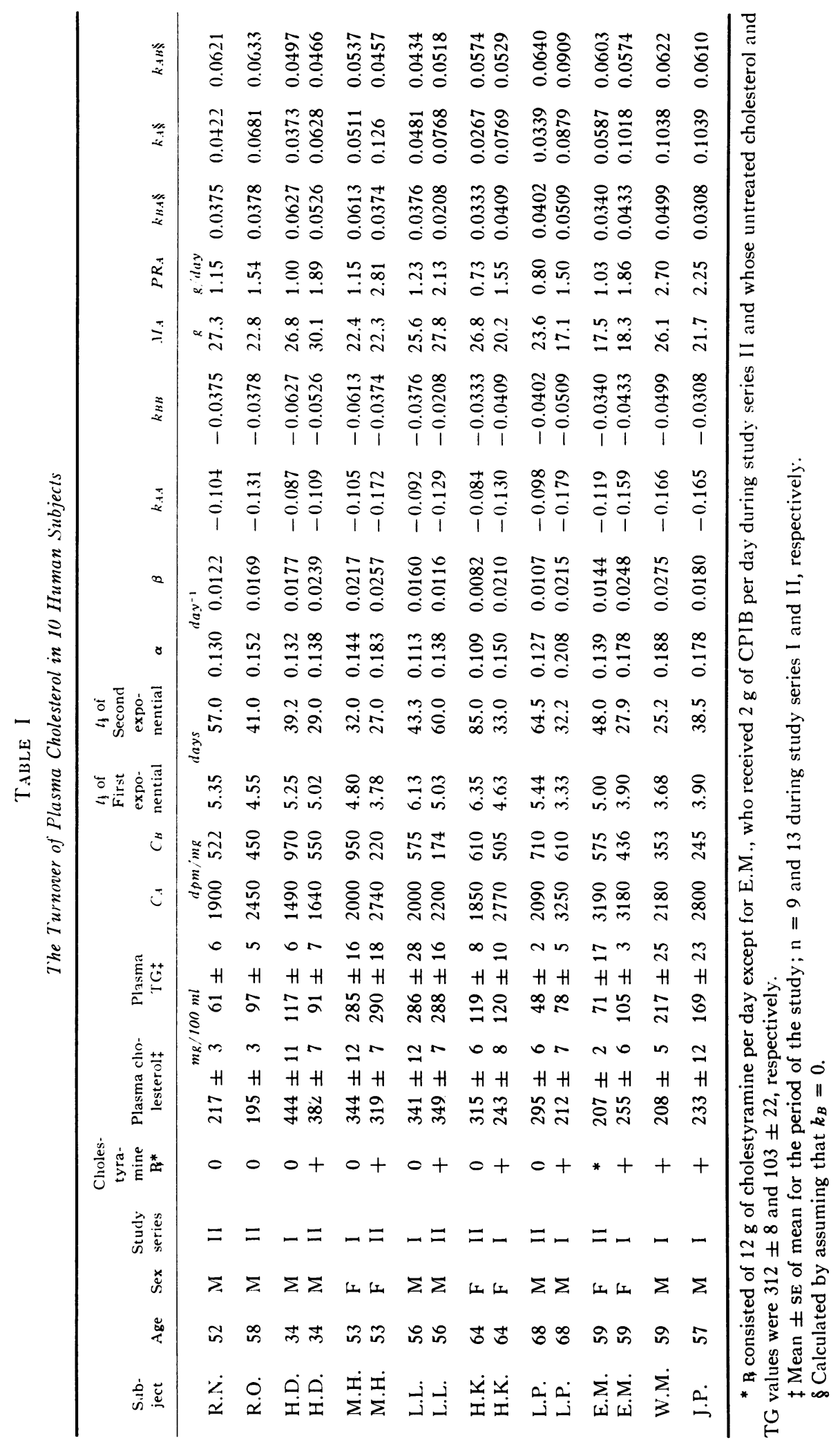


TABLE II

Turnover Parameters as Determined by Computer Analysis*

\begin{tabular}{|c|c|c|c|c|c|c|}
\hline $\begin{array}{l}\text { Patient........ } \\
\mathbf{R} \ldots \ldots \ldots \ldots \ldots\end{array}$ & $\begin{array}{l}\ldots \ldots \text { H.D. } \\
\ldots \ldots \ldots \ldots \text {. }\end{array}$ & $\begin{array}{l}\text { H.D. } \\
+\end{array}$ & $\begin{array}{l}\text { L.L. } \\
0\end{array}$ & $\begin{array}{l}\text { L.L. } \\
+\end{array}$ & $\begin{array}{l}\text { L.P. } \\
0\end{array}$ & $\begin{array}{l}\text { L.P. } \\
+\end{array}$ \\
\hline$C_{A}, d p m / m g$ & $1499 \pm 5.9 \%$ & $1682 \pm 3.6 \%$ & $1970 \pm 2.6 \%$ & $2129 \pm 3.0 \%$ & $2091 \pm$ & $3356 \pm 1.8 \%$ \\
\hline$C_{B}, d p m / m g$ & $950 \pm 9.8 \%$ & $504 \pm 8.5 \%$ & $551 \pm 9.8 \%$ & $198 \pm 9.0 \%$ & $684 \pm$ & $614 \pm 2.6 \%$ \\
\hline$\alpha, d a y^{-1}$ & $0.128 \pm 12.8 \%$ & $0.134 \pm 6.7 \%$ & $0.108 \pm 5.1 \%$ & $0.144 \pm 3.8 \%$ & $0.124 \pm 5.6 \%$ & $0.218 \pm 2.5 \%$ \\
\hline$\beta, d a y^{-1}$ & $0.0176 \pm 10.0 \%$ & $0.0220 \pm 6.6 \%$ & $0.0161 \pm 10.2 \%$ & $0.0134 \pm 12.0 \%$ & $0.0101 \pm 10.3 \%$ & $0.0215 \pm 2.5 \%$ \\
\hline$k_{B A}$ & $0.0600 \pm 19.0 \%$ & $0.0480 \pm 10.7 \%$ & $0.0364 \pm 12.5 \%$ & $0.0248 \pm 11.5 \%$ & $0.0386 \pm 10.1 \%$ & $0.0515 \pm 3.6 \%$ \\
\hline$k_{A}$ & $0.0373 \pm 5.4 \%$ & $0.0616 \pm 3.5 \%$ & $0.0481 \pm 3.8 \%$ & $0.0791 \pm 4.6 \%$ & $0.0330 \pm 6.0 \%$ & $0.0900 \pm 1.9 \%$ \\
\hline$k_{A B}$ & $0.0476 \pm 14.3 \%$ & $0.0468 \pm 9.3 \%$ & $0.0399 \pm 4.8 \%$ & $0.0539 \pm 4.2 \%$ & $0.0633 \pm 5.4 \%$ & $0.0965 \pm 3.4 \%$ \\
\hline
\end{tabular}

* The values listed represent the best value for each parameter, as determined by a least squares solution, together with the standard deviation for each parameter. The values of $C_{A}, C_{B}, \alpha$, and $\beta$ were determined by analyzing the data in terms of a two-pool exponential as described by equation (1). The rate constants $\left(k_{B A}, k_{A}\right.$, and $\left.k_{A B}\right)$ were calculated independently of the values obtained for $C_{A}, C_{B}, \alpha$, and $\beta$, by using a two-compartment model in which $k_{B}=0$.

tients in whom administration of cholestyramine at a dose of $12 \mathrm{~g} /$ day produced an average decrease of $13 \%$ in the plasma cholesterol level, whereas a dose of $24 \mathrm{~g}$ /day produced an average decrease of $20 \%$ (R. P. Noble, unpublished observations).

Table III summarizes the results obtained without therapy and during cholestyramine therapy in this group of five patients. The most significant effects of cholestyramine therapy were: (a) a $100 \%$ increase in the production rate of cholesterol in pool $\mathrm{A} ;(b)$ at $54 \%$ increase in the rate of removal of cholesterol from pool A $\left(\mathrm{k}_{\boldsymbol{A} A}\right)$; and $(c)$ a great increase in $k_{\mathbf{A}}$. Cholestyramine therapy did not significantly alter the size of pool A, but did result in a decrease in the half-lives of both exponentials. The latter effect was statistically probably significant $(0.02<P<0.05)$ for the first

TABLE III

Mean Results of Cholesterol Turnover Studies

\begin{tabular}{|c|c|c|c|c|}
\hline & \multicolumn{2}{|c|}{5 Patients (mean \pm SEM) } & \multirow{2}{*}{$\begin{array}{c}\text { Effect of } \\
\text { cholestyramine } \\
\mathbf{R}^{*}\end{array}$} & \multirow{2}{*}{$\begin{array}{l}2 \text { Normal } \\
\text { controls } \\
\text { (mean) }\end{array}$} \\
\hline & No $R$ & Cholestryamine $R$ & & \\
\hline$C_{A}, d p m / m g$ & $1886 \pm 106$ & $2520 \pm 276$ & PS & 2175 \\
\hline$C_{B}, d p m / m g$ & $763 \pm 83$ & $412 \pm 90$ & PS & 486 \\
\hline$t_{\frac{1}{}}$ first exponential, days & $5.59 \pm 0.29$ & $4.36 \pm 0.34$ & PS & 4.95 \\
\hline$t_{\mathbf{b}}$ second exponential, days & $52.8 \pm 9.7$ & $36.2 \pm 6.0$ & $\mathrm{NS}(>0.1)$ & 49.0 \\
\hline$\alpha, d a y^{-1}$ & $0.1252 \pm 0.0064$ & $0.1634 \pm 0.0139$ & PS & 0.1410 \\
\hline$\beta, d a y^{-1}$ & $0.0148 \pm 0.0024$ & $0.0207 \pm 0.0024$ & $\mathrm{NS}(>0.1)$ & 0.0146 \\
\hline$k_{A A}$ & $-0.0931 \pm 0.0037$ & $-0.1437 \pm 0.0134$ & SS & -0.1179 \\
\hline$k_{B B}$ & $-0.0470 \pm 0.0062$ & $-0.0405 \pm 0.0057$ & $\mathrm{NS}(>0.4)$ & -0.0377 \\
\hline$M_{A}, g$ & $25.05 \pm 0.89$ & $23.50 \pm 2.41$ & $\mathrm{NS}(>0.5)$ & 25.01 \\
\hline$P R_{A}, \mathrm{~g} /$ day & $0.98 \pm 0.10$ & $1.98 \pm 0.24$ & SS & 1.35 \\
\hline$k_{B A} t$ & $0.0470 \pm 0.0062$ & $0.0405 \pm 0.0057$ & $\mathrm{NS}(>0.4)$ & 0.0377 \\
\hline$k_{A} t$ & $0.0394 \pm 0.0045$ & $0.0861 \pm 0.0107$ & SS & 0.0552 \\
\hline$k_{A B} \ddagger$ & $0.0536 \pm 0.0035$ & $0.0576 \pm 0.0084$ & $\mathrm{NS}(>0.6)$ & 0.0627 \\
\hline
\end{tabular}

* The effect of cholestyramine therapy on each parameter was evaluated by determining the probability $(P)$ that the results obtained during therapy differed from those obtained without therapy. The results obtained with and without cholestyramine were paired for each subject, and the five difference values so obtained were statistically compared to zero, by means of Student's $t$ test, using a two-tailed $t$ test with 4 degrees of freedom (18). NS means that the effect of therapy was not significant ( $P$ values given in parentheses). PS means that the effect of therapy was statistically probably significant $(0.01<P<0.05)$, and SS means that the effect was statistically significant $(P<0.01)$.

$\ddagger$ Calculated by assuming that $k_{B}=0$. 


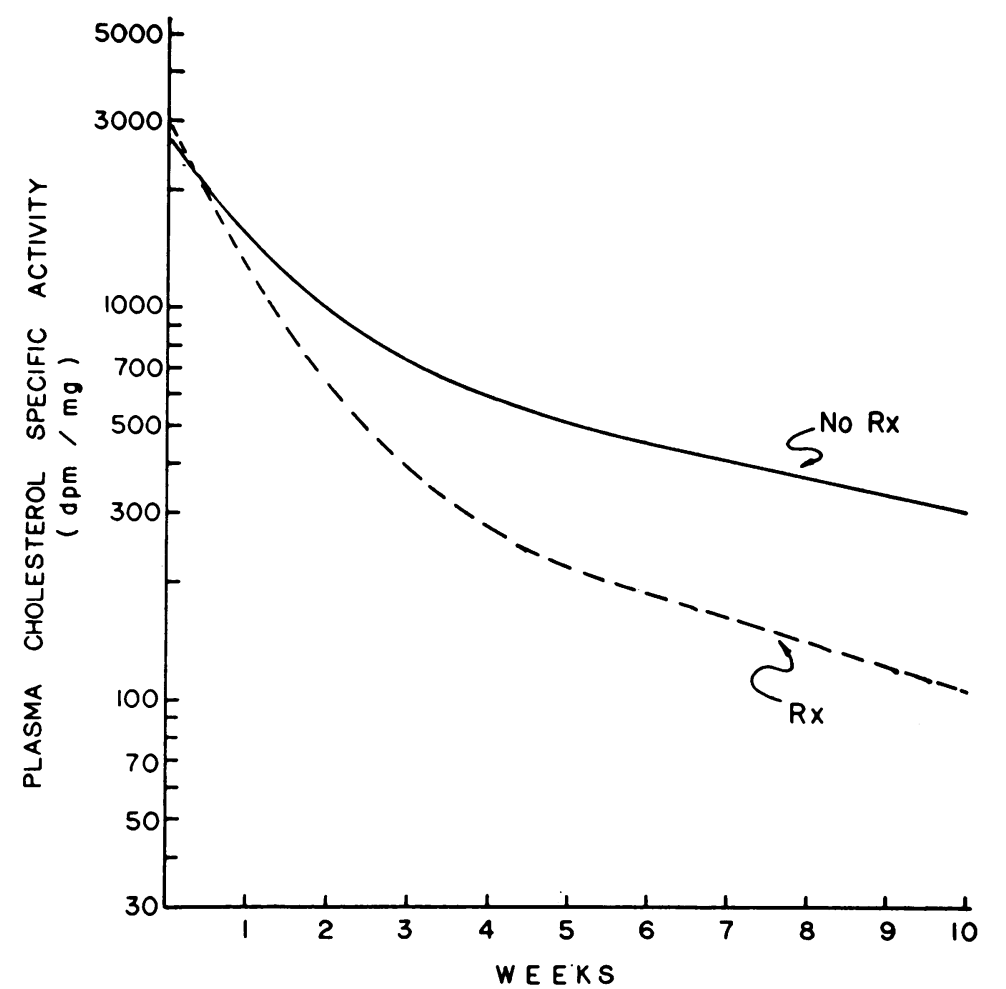

Figure 3 The effect of cholestyramine on the turnover of plasma cholesterol. The curves shown are the mean plasma turnover curves, constructed from the values given in Table III, for the five subjects who were studied both off and on cholestyraminc therapy. exponential, but was not statistically significant $(0.1<P<0.2)$ for the second exponential. ${ }^{1}$

The effect of cholestyramine on the turnover of plasma cholesterol is shown graphically in Fig. 3, which presents the mean curves obtained both off and on therapy for the five subjects studied under both conditions.

One subject. (E.M.) was studied first during a period of cholestyramine therapy and subsequently while being treated with CPIB. In this

1 Additional information about the kinetic parameters involved in the model for cholesterol turnover and about the effect of cholestyramine can be obtained by postulating that the change produced in the system by therapy is limited to one or two parameters. This approach to the effect of a "perturbation" on a model has been discussed by Berman (19). If, for example, one assumes that cholestyramine therapy does not alter the values of the rate constants $k_{A B}, k_{B A}$, or $k_{B}$, it is possible to obtain ranges for the values of all the rate constants shown in Fig. 2 $\left(k_{A B}, k_{B A}, k_{A}\right.$, and $\left.k_{B}\right)$ for the complete model where $k_{B}$ is not assumed to equal zero. Computer analysis (kindly carried out by Dr. Frank) of the data of subjects H.D., L.L., and L.P. indicated that the data were consistent with a model which assumes that $\mathrm{k}_{\mathbf{B}}$ equals zero and that cholestyramine therapy does not alter the values of $k_{A B}$ and kBA. subject, the results (Table I) obtained during CPIB therapy appeared similar to results obtained in the untreated subjects, when compared to the results obtained during cholestyramine therapy. No conclusions about the effects of CPIB therapy can be derived from this one study. The study is included here merely to indicate that during CPIB as well as during cholestyramine therapy the turnover of plasma cholesterol conformed to a two-pool model. More extensive studies on the effect of CPIB on cholesterol turnover were recently reported by Nestel, Hirsch, and Couzens (6).

Normal vs. hyperlipidemic subjects. Table III also presents the mean values obtained with the two normal control subjects (R.N. and R.O.). Comparison of these values with the values obtained without treatment for the five hyperlipidemic subjects demonstrates that the size of pool A and the half-lives of the two exponentials were very similar in the normal and the hyperlipidemic subjects. Because of the small number of normal subjects studied it is not possible to evaluate properly the possibility that some of the other parameters might differ significantly for normal as com- 
pared to hyperlipidemic subjects. With the data on hand, the only parameter which was significantly different for the normal as compared to the hyperlipidemic subjects was $\mathrm{C}_{\mathbf{B}} \quad(0.02<P<$ $0.05)$.

\section{DISCUSSION}

In the studies reported here, compartmental analysis of the turnover curves of plasma cholesterol indicated that the turnover of plasma cholesterol in man can be described by a two-pool model. This was true for every one of the 16 studies which we have conducted in normal men, in untreated hyperlipidemic patients, and in hyperlipidemic patients being treated with cholestyramine or CPIB. It is of interest that Avigan, Steinberg, and Berman likewise observed that the turnover of serum cholesterol in the rat could be described by an equation for a two-compartment system (20). In contrast, Casdorph et al. reported that the turnover of plasma cholesterol in dogs was best fitted by a four-compartment system (21).

In discussing the turnover of plasma cholesterol in terms of a two-pool model, it must be clearly recognized that the pools in question (see Fig. 2) represent mathematical constructs and do not have precise physical meaning. It is now well established that the in vivo turnover of cholesterol in man involves a large number of metabolically heterogeneous pools of cholesterol in different tissues and within given tissues $(8,9,22,23)$. Even within plasma itself, four metabolically heterogeneous pools of cholesterol (free cholesterol, and ester cholesterol in each of three plasma lipoprotein fractions) have been distinguished (23). The finding that the turnover of plasma total cholesterol conforms to a two-pool model hence means that the various tissue pools of cholesterol fall into two groups in terms of the rates at which they equilibrate with plasma cholesterol. One group of pools is apparently in fairly rapid equilibrium (in terms of hours to days) with plasma cholesterol, whereas the second group of pools is in fairly slow equilibrium (in terms of days to weeks) with plasma cholesterol. Within each group, the rates of equilibration of the different pools with plasma cholesterol are apparently sufficiently similar so that the group behaves as a single pool, when analyzed in terms of the turnover curve of plasma total cholesterol. In the present studies, all of the data, for samples collected from $8 \mathrm{hr}$ to $10 \mathrm{wk}$ after isotope injection, conformed precisely to a two-pool model. It is of course, possible that samples collected during the first 8 $\mathrm{hr}$ might have revealed the presence of an additional kinetically distinguishable compartment.

Information available in the literature permits some inferences to be drawn about the tissue locations of the cholesterol molecules which comprise the two compartments (pools A and B) shown in Fig. 2. It is well established that plasma free cholesterol, plasma esterified cholesterol, red blood cell cholesterol, and liver cholesterol all equilibrate with each other quite rapidly $(8,9$, 22-26). It seems fairly certain, therefore, that plasma, red cell, and liver cholesterol, together comprising approximately $15 \mathrm{~g}$ of cholesterol in the average adult, are all part of the rapidly turning over pool (pool A). In addition, it is likely that some of the cholesterol in several other viscera [e.g., spleen, kidney, lung, intestines (9)] also equilibrates with plasma cholesterol sufficiently fast to comprise part of pool A. The remainder of the cholesterol in these viscera, together with most of the cholesterol in peripheral tissues [particularly skeletal muscle $(8,9)$ ], equilibrates more slowly with plasma cholesterol and comprises the more slowly turning over pool, pool B.

A significant, albeit linited, amount of information about cholesterol metabolism can be determined by analyzing plasma cholesterol turnover in terms of the general two-pool model (Fig. 2). As cliscussed above, the parameters which can be determined include the constants $C_{A}, C_{B}$, $\alpha$, and $\beta$ (see equation 1 ), the size of the first pool $\left(M_{A}\right)$, the rate constants for the total rate of removal of cholesterol from each pool $\left(\mathrm{k}_{\mathbf{A A}}\right.$ and $\left.\mathrm{k}_{\mathrm{BB}}\right)$, and the production rate in pool $A\left(P R_{\mathbf{A}}\right)$ (see reference 13 for complete discussion). An important parameter which cannot be derived from the plasma cholesterol turnover curve is the size of the second pool, pool B. Gurpide et al. (13) have indicated that the size of the second pool can only be determined when either $s_{A}$ and $k_{A}$ or $s_{B}$ and $k_{B}$ both equal zero. Since the liver and the intestine are the major organs involved in cholesterol biosynthesis, it is certain that $\mathbf{s}_{\mathbf{A}}$ does not equal zero. It is also highly unlikely that $\mathrm{s}_{\mathrm{B}}$ equals zero, since it is well established that virtually all organs and tissues are capable of carrying out cholesterol biosynthesis 
(27-29). The special conditions which may permit the calculation of the size of the second pool therefore do not apply to cholesterol turnover. Accordingly, it is not possible to calculate the size of the total body exchangeable pool of cholesterol from the turnover curve of plasma cholesterol.

Another parameter which cannot be determined in the general two-pool model (Fig. 2) is the metabolic turnover rate of cholesterol (that is, the rate of cholesterol degradation and excretion) in the whole body. Several previous studies of cholesterol turnover in man have estimated the total metabolic turnover rate of cholesterol by assuming that the late, linear portion of the plasma turnover curve represents the half-life of the total body miscible cholesterol. This procedure is not correct $(13,30)$ since the slope of the late, linear portion of the turnover curve (a measure of $\beta$ in equation 1) has no precise physical meaning and is a complex mathematical function of all of the rate constants shown in Fig. 2.2 The metabolic turnover rate of cholesterol can, however, be calculated by assuming that $k_{B}$, the rate of removal of cholesterol from the system directly via pool B, approximates zero. This assumption seems reasonable since there is probably only a quantitatively unimportant amount of cholesterol degradation and excretion directly via the peripheral tissue and organ pools which comprise pool B. The known quantitatively important pathways of cholesterol metabolism include the catabolism of cholesterol to bile acids in the liver and the excretion of acidic steroids and neutral sterols via the feces $(7,31-$ $33)$. The fecal sterols and sterol metabolites originate mainly in the liver and also partly in the intestinal mucosa. It has also been suggested recently that a variable amount of sterol ring degradation and loss occurs in the intestinal lumen (34). It seems likely that these pathways of cholesterol degradation and excretion originate almost completely in tissue pools which comprise pool A.

As noted above, by assuming that $k_{B}$ equals zero it is possible to calculate $k_{\mathbf{A}}, \mathrm{k}_{\mathbf{B} \mathbf{A}}$, and $\mathrm{k}_{\mathbf{A B}}$. By combining $k_{\mathbf{A}}$, the rate constant for the direct

\footnotetext{
2 The constants $\alpha$ and $\beta$ can be expressed as quadratic functions of the rate constants $k_{A A}, k_{B B}, k_{A B}$, and $k_{B A}$. These functions can readily be derived from the following expressions: $\alpha+\beta=-\left(\mathrm{k}_{\mathrm{AA}}+\mathrm{k}_{\mathrm{BB}}\right) ;$ and $\alpha \beta=$ $k_{A A} k_{B B}-k_{A B} k_{B A}$.
}

removal of cholesterol from pool $\mathrm{A}$ (and hence from the system, since $k_{B}=0$ ), with the size of pool A, the metabolic turnover of cholesterol from the body, in grams per day, can be calculated readily. This calculation can be omitted, however, since it is evident from inspection of Fig. 2 that the rate of cholesterol entry into and loss from the system (i.e., the metabolic turnover rate) is identical with the production rate in pool $\mathrm{A}$ for the special case where $k_{B}$ equals zero. This is, of course, true only in the steady state. The production rate in pool A, calculated according to equation (5), hence should provide a valid measurement of the rate of cholesterol degradation and excretion in the whole body. The validity of this interpretation of $\mathrm{PR}_{\mathbf{A}}$ depends, of course, on the correctness of the assumption that $k_{B}$ equals zero. Future studies, comparing the production rate with the rate of cholesterol turnover as measured by total balance methods, will be necessary in order to determine whether or not the production rate provides a valid estimate of the metabolic turnover rate of cholesterol.

The studies presented here illustrate the information which can be obtained by a two-pool compartmental analysis of the turnover of plasma cholesterol in man. In both normal and hyperlipidemic subjects, the first pool (pool A) was found to contain approximately $25 \mathrm{~g}$ of cholesterol, and the values of $\alpha$ and $\beta$ were found to be approximately 0.13 and 0.015 , respectively. The mean value for the production rate in pool $\mathrm{A}$ was 0.98 $\mathrm{g} /$ day for the hyperlipidemic and $1.35 \mathrm{~g} /$ day for the normal subjects. Treatment with cholestyramine minimally affected the size of pool A, decreased the half-lives of both exponentials to a small extent, and greatly increased the production rate in pool A and the rate of removal of cholesterol from pool A. If we assume that the production rate provides a valid estimate of the metabolic turnover rate, then these results indicate that cholestyramine produces a $100 \%$ increase in the rate of cholesterol degradation and excretion from the body.

\section{ACKNOWLEDGMENTS}

We are grateful for the expert assistance of $\mathrm{Mr}$. T. Shiratori, Miss Grace Stanton, and Mrs. Joan Vormbaum. We particularly wish to thank Dr. E. Gurpide for stimulating and illuminating advice and discussions. We are very grateful to Doctors A. Frank, D. Steinberg, 
and M. Berman for the computer analysis of some of the data.

This work was supported by Research Grant AM05968 from the National Institutes of Health, Bethesda, Md., and by grants from the Sharon Research Institute and from Mead Johnson \& Co. Dr. Goodman is a recipient of a Career Scientist Award from the Health Research Council of the City of New York under Contract 1-399.

\section{REFERENCES}

1. Goodman, D. S., and R. P. Noble. 1966. Cholesterol ester turnover in human plasma lipoproteins during cholestyramine therapy. Circulation. 34 (Suppl. 3) : 12 (Abstr.)

2. Hellman, L., R. S. Rosenfeld, and T. F. Gallagher. 1954. Cholesterol synthesis from $\mathrm{C}^{14}$-acetate in man. J. Clin. Invest. 33: 142.

3. Kurland, G. S., J. L. Lucas, and A. S. Freedberg. 1961. The metabolism of intravenously infused $C^{14}-$ labeled cholesterol in euthyroidism and myxedema. J. Lab. Clin. Med. 57: 574.

4. Chobanian, A. V., B. A. Burrows, and W. Hollander. 1962. Body cholesterol metabolism in man. II. Measurement of the body cholesterol miscible pool and turnover rate. J. Clin. Invest. 41: 1738.

5. Gidez, L. I., and H. A. Eder. 1963. Cholesterol turnover in man. In Effects of Drugs on Synthesis and Mobilization of Lipids. Proceedings of the First International Pharmacological Meeting, 1961. E. C. Horning and $\mathrm{P}$. Lindgren, editors. Pergamon Press Ltd., Oxford, 2: 67.

6. Nestel, P. J., E. Z. Hirsch, and E. A. Couzens. 1965. The effect of chlorophenoxyisobutyric acid and ethinyl estradiol on cholesterol turnover. J. Clin. Invest. 44: 891.

7. Grundy, S. M., and E. H. Ahrens, Jr. 1966. An evaluation of the relative merits of two methods for measuring the balance of sterols in man: isotopic balance versus chromatographic analysis. J. Clin. Invest. 45: 1503.

8. Field, H., Jr., L. Swell, P. E. Schools, Jr., and C. R. Treadwell. 1960. Dynamic aspects of cholesterol metabolism in different areas of the aorta and other tissues in man and their relationship to atherosclerosis. Circulation. 22: 547.

9. Chobanian, A. V., and W. Hollander. 1962. Body cholesterol metabolism in man. I. The equilibration of serum and tissue cholesterol. J. Clin. Invest. 41: 1732.

10. Abell, L. L., B. B. Levy, B. B. Brodie, and F. E. Kendall. 1952. A simplified method for the estimation of total cholesterol in serum and demonstration of its specificity. J. Biol. Chem 105: 357 .

11. Van Hande1, E., and D. B. Zilversmit. 1957. Micromethod for the direct determination of serum triglycerides. J. Lab. Clin. Med. 50: 152.

12. Berman, M. 1963. The formulation and testing of models. Ann. N. Y. Acad. Sci. 108: 182.
13. Gurpide, E., J. Manr., and E. Sandberg. 1964. Determination of kinetic parameters in a two-pool system by administration of one or more tracers. Biochemistry. 3: 1250.

14. Gurpide, E., J. Mann, and S. Lieberman. 1963. Analysis of open systems of multiple pools by administration of tracers at a constant rate or as a single dose as illustrated by problems involving steroid hormones. J. Clin. Endocrinol. Metab. 23: 1155.

15. Berman, M., E. Shahn, and M. F. Weiss. 1962. The routine fitting of kinetic data to models: a mathematical formalism for digital computers. Biophys. J. 2: 275 .

16. Berman, M., M. F. Weiss, and E. Shahr. 1962. Some formal approaches to the analysis of kinetic data in terms of linear compartmental systems. Biophys. J. 2: 289.

17. Berman, M. Compartmental analysis in kinetics. 1965. In Computers in Biomedical Research. R. W. Stacy and B. D. Waxman, editors. Academic Press Inc., New York. 2: 173.

18. Dixon, W. J., and F. J. Massey, Jr. 1957. Introduction to statistical analysis. McGraw-Hill Book Company, New York. 2nd edition.

19. Berman, M. 1963. A postulate to aid in model building. J. Theoret. Biol. 4 : 229.

20. Avigan, J., D. Steinberg, and M. Berman. 1962. Distribution of labeled cholesterol in animal tissues. J. Lipid Res. 3: 216.

21. Casdorph, H. R., J. L. Juergens, A. L. Orvis, and C. A. Owen, Jr. 1963. Rate of disappearance of cholesterol-C-14 from the bloodstream of dogs. Proc. Soc. Exptl. Biol. Med. 112: 191.

22. Goodman, D. S. 1965. Cholesterol ester metabolism. Physiol. Rev. 45 : 747.

23. Goodman, D. S. 1964. The in vivo turnover of individual cholesterol esters in human plasma lipoproteins. J. Clin. Invest. 43: 2026.

24. Gould, R. G., G. V. LeRoy, G. T. Okita, J. J. Kabara, P. Keegan, and D. M. Bergenstal. 1955. The use of $\mathrm{C}^{14}$-labeled acetate to study cholesterol metabolism in man. J. Lab. Clin. Med. 46: 372.

25. Gould, R. G., and R. P. Cook. 1958. The metabolism of cholesterol and other sterols in the animal organism. In Cholesterol. R. P. Cook, editor. Academic Press Inc., New York. 237.

26. Cox, G. E., C. B. Taylor, D. Patton, C. Davis, Jr., and N. Blandin. 1963. Origin of plasma cholesterol in man. Arch. Pathol. 76: 60.

27. Srere, P. A., I. L. Chaikoff, S. S. Treitman, and L. S. Burstein. 1950. The extrahepatic synthesis of cholesterol. J. Biol. Chem. 182: 629.

28. Eckles, N. E., C. B. Taylor, D. J. Campbell, and R. G. Gould. 1955. The origin of plasma cholesterol and the rates of equilibration of liver, plasma, and erythrocyte cholesterol. J. Lab. Clin. Med. 46: 359.

29. Dietschy, J. M., and M. D. Siperstein. 1967. Effect of cholesterol feeding and fasting on sterol synthesis in seventeen tissues of the rat. J. Lipid Res. 8: 97. 
30. Tait, J. F., S. A. S. Tait, B. Little, and K. R. Laumas. 1961. The disappearance of $7-\mathrm{H}^{3}-\mathrm{d}$-aldosterone in the plasma of normal subjects. J. Clin. Invest. 40: 72.

31. Spritz, N., E. H. Ahrens, Jr., and S. Grundy. 1965. Sterol balance in man as plasma cholesterol concentrations are altered by exchanges of dietary fats. J. Clin. Invest. 44: 1482.

32. Wilson, J. D., and C. A. Lindsey, Jr. 1965. Studies on the influence of dietary cholesterol on cholesterol metabolism in the isotopic steady state in man. J. Clin. Invest. 44: 1805.

33. Avigan, J., and D. Steinberg. 1965. Sterol and bile acid excretion in man and the effects of dietary fat. J. Clin. Invest. 44: 1845.

34. Ahrens, E. H., Jr. 1966. Degradation of the sterol ring structure in the intestine, and the significance of this for sterol balance studies. Gastroenterology. 51 : 596. (Abstr.) 